\title{
A novel mutation in PAX9 causes familial form of molar oligodontia
}

\author{
Adrianna Mostowska*,1, Barbara Biedziak ${ }^{2}$ and Wieslaw H Trzeciak ${ }^{1}$ \\ ${ }^{1}$ Department of Biochemistry and Molecular Biology, University of Medical Sciences, Poznan, Poland; ${ }^{2}$ Department \\ of Orthodontics, University of Medical Sciences, Poznan, Poland
}

PAX9 is a paired domain transcription factor that plays a critical role in odontogenesis. All mutations of PAX9 identified to date have been associated with nonsyndromic form of tooth agenesis. The present report describes an unusual novel mutation in PAX9 identified in a family with severe molar oligodontia. This heterozygous deletion combined with $24 \mathrm{bp}$ insertion (including a $5^{\prime}$ splice site) is localized in the second exon beyond the highly conserved paired box sequence, and might result either in a premature termination of translation at aa $\mathbf{2 1 0}$ or in an aberrant splicing, leading to a frameshift and premature termination of translation at aa 314. Real-time PCR analysis revealed no mutated transcript in cultured lymphocytes of one of the affected individuals indicating that the novel mutation might result in rapid degradation of the mutated transcript leading to haploinsufficiency of PAX9. Our results support the view that mutations in PAX9 constitute a causative factor in nonsyndromic oligodontia.

European Journal of Human Genetics (2006) 14, 173-179. doi:10.1038/sj.ejhg.5201536; published online 7 December 2005

Keywords: oligodontia; PAX9; mutation; mRNA degradation

\section{Introduction}

Agenesis of permanent teeth constitutes one of the most common developmental abnormalities in humans (OMIM: \#106600, \#604625). Tooth agenesis most commonly involves third molars, and affects $10-25 \%$ of the population, whereas the overall incidence of missing permanent teeth, excluding third molars, varies from 2 to $10 \%{ }^{1}$ To describe dental anomalies, several different terms are being used. Hypodontia is defined as an absence of one to six permanent teeth, excluding third molars, whereas the absence of more than six teeth is referred to as oligodontia. The most extreme case is anodontia denoting absence of all teeth. ${ }^{2}$

It has been demonstrated that nonsyndromic form of familial and sporadic tooth agenesis is associated with mutations in MSX1 and PAX9. ${ }^{3-16}$ Protein products of

*Correspondence: Dr A Mostowska, Department of Biochemistry and Molecular Biology, University of Medical Sciences, Swiecickiego St. 6, 60781 Poznan, Poland. Tel: + 4861854 6515; Fax: + 4861854 6513; E-mail: amostowska@wp.pl

Received 21 June 2005; revised 4 October 2005; accepted 14 October 2005; published online 7 December 2005 these genes serve as transcription factors that play a critical role during embryogenesis. ${ }^{17,18}$ Mice homozygous for Ms 1 or Pax9 deletion exhibit secondary cleft palate, facial abnormalities and complete anodontia, with concomitant arrest of tooth development at the bud stage. ${ }^{19,20}$

It is noteworthy that in humans all mutations of the genes responsible for selective tooth agenesis were found in heterozygous state, suggesting that lack of teeth in the affected individuals was likely due to a loss of function of a single allele leading to haploinsufficiency. ${ }^{21,22}$ In addition, these mutations caused only familial forms of oligodontia, with the exception of c. $151 \mathrm{G}>\mathrm{A}$ transition in PAX9 identified in Polish population, the first de novo mutation described in this gene. ${ }^{13}$

Most recent studies indicate that a novel candidate gene for selective tooth agenesis is AXIN2 located on chromosome $17 q 23-q 24$. Protein product of this gene plays an important role in the regulation of beta-catenin stability in signal transduction via the Wnt signaling pathway, involved in regulation of embryonic patterning, stem cell renewal and cancer. ${ }^{23}$ Although mutations of AXIN2 have 
previously been associated with colorectal carcinomas and liver tumors, recent studies indicate that they might also be responsible for the lack of teeth. ${ }^{24,25}$

There is also evidence that in the Brazilian population, polymorphisms of the gene encoding transforming growth factor alpha $(T G F \alpha)$ are associated with isolated form of this common developmental anomaly, especially when tooth agenesis includes incisors. ${ }^{26}$

In the present report, a novel mutation of PAX9 was described in a three-generation family with severe oligodontia. This mutation might be a cause of rapid degradation of PAX9 mRNA.

\section{Materials and methods}

Family with oligodontia and control samples

A female proband was a patient of the Department of Orthodontics, University of Medical Sciences in Poznan. The diagnosis of oligodontia was based on clinical examination, panoramic radiograph and an interview. All her family members (four affected and five unaffected) were also clinically examined, and the lack of permanent teeth was verified with panoramic radiographs. In addition, 150 healthy individuals who did not exhibit any abnormalities in either the number or the shape of teeth were used as controls. The study protocol was approved by the local Ethics Committee and written consents from the patients were obtained.

PAX9 and MSX1 mutation screening and sequencing Genomic DNA was isolated from peripheral blood lymphocytes by salt extraction. The coding region of $P A X 9$ was amplified with the use of six sets of primers (sequences available on request). Some of the primers were previously described. ${ }^{11}$ The coding region of MSX1 was amplified with the use of primers described by Lidral et al. ${ }^{27}$ In order to screen for mutations, the amplified fragments were subjected to multi-temperature, single-stranded conformational polymorphism analysis (MSSCP), with the use of a DNA Pointer Mutation Detection System (Kucharczyk T.E., Warsaw, Poland). In addition, the amplified fragment PAX9ex2C was subjected to $10 \%$ polyacrylamide gel electrophoresis (PAGE). Two DNA bands, observed during PAGE analysis in the samples derived from the patients with tooth agenesis, were excised from the gel and reamplified. To establish the localization and the character of the mutation, the amplified or reamplified fragments were purified by the GelOut system (A\&A Biotechnology, Warsaw, Poland) and sequenced using ABI Prism BigDye Terminator v3.1 Cycle Sequencing Kit and ABI 310 genetic analyzer (Applied Biosystems, Foster City, CA, USA).

\section{Restriction analysis}

Genomic DNA of all family members was amplified with specific primers MSXUTRF 5'-ACAAGAGGCAGAGCTGGA
GAA-3' and MSXUTRR 5'-GAAACTGGAGCAGTGTGAG GGT-3' for MSX1 and PAXex3F 5'-GGGAGTAAAACTTCAC CAGGC-3' and PAXex3R 5'-CCACCTGGCCTGACCCTC-3' for $P A X 9$. The amplified fragments were then subjected to restriction digestion with DraII (PuG/GNCCPy) and MspI (C/CGG), respectively (Fermentas, Vilnius, Lithuania) according to the manufacturer's instructions. Digestion products were analyzed by agarose gel electrophoresis.

\section{Analysis of PAX9 cDNA}

Peripheral blood lymphocytes of the proband were isolated by centrifugation in a ficol gradient $\left(1077 \mathrm{~g} / \mathrm{cm}^{3}\right)$, cultured in RPMI 1640 medium supplemented with L-glutamine and fetal bovine serum, and stimulated with PHA $(10 \mu \mathrm{g} /$ $\mathrm{ml})$ and IL-2 (100 U/ml). Total RNA was extracted with Trizol (Gibco BRL, Germany) according to the manufacturer's instructions. RNA was digested for $30 \mathrm{~min}$ at $37^{\circ} \mathrm{C}$ with RQ1 RNase-free DNase (Promega, USA), re-extracted with Trizol and reverse transcribed (RT-PCR) with the use of Enhanced Avian HS RT-PCR Kit (Sigma, USA). RNA isolated from JURKAT cells, a cell line derived from human T-cell leukemia was used as a control. The cDNA amplification was performed with the use of following primers: PAXCDNAF 5'-GCTGCCCTACAACCACATCT-3', PAXCDNAR 5'GTACTTGGCTTCCT GCTCCA-3' and $\beta$ actF 5' GCACCA CACCTTCTACAATGAGC-3', $\beta$ actR 5'- GGATAGCACAGCC TGGATAGCAAC- $3^{\prime}$. The conditions for the PCR were $95^{\circ} \mathrm{C}$ for $5 \mathrm{~min}, 94^{\circ} \mathrm{C}$ for $30 \mathrm{~s}, 60^{\circ} \mathrm{C}$ for $35 \mathrm{~s}, 72^{\circ} \mathrm{C}$ for $35 \mathrm{~s}$, with a final extension of $5 \mathrm{~min}$ after 35 cycles. The amplification products were analyzed by electrophoresis in 10\% PAA, purified and sequenced using ABI Prism BigDye Terminator v3.1 Cycle Sequencing Kit and ABI 310 genetic analyzer (Applied Biosystems, Foster City, CA, USA). The cDNA region spanning the second and the third exon of PAX9 was also analyzed by real-time PCR with the use of the LightCycler System and LightCycler FastStart DNA Master SYBR Green Kit (Roche Diagnostics GmbH, Mannheim, Germany) according to the manufacturer's instructions. The amplification was performed with the use of following primers: PAXcDNA1F: 5'-ACTCCGTCACCGACATCCT-3' and PAXcDNA1R: 5'-CTTC TCCAACCCGTTCACC- $3^{\prime}$. The annealing temperature was $60^{\circ} \mathrm{C}$. The PCR products were identified by generating a melting curve, which was then redrawn as the negative derivative of fluorescence with respect to temperature in order to generate a melting peak.

\section{Results}

\section{Genetic and clinical examination}

We analyzed nine members of a three generation family with oligodontia segregated as an autosomal dominant trait (Figure 1a). Clinical and radiographic examinations revealed that proband (III:1) and four other members of her family (I:1, II:1, II:4 and III:2) lacked 18 permanent teeth. 
a

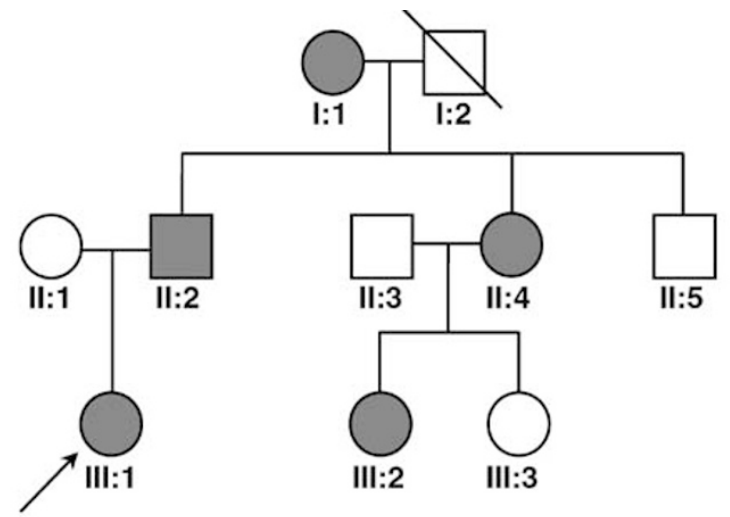

b

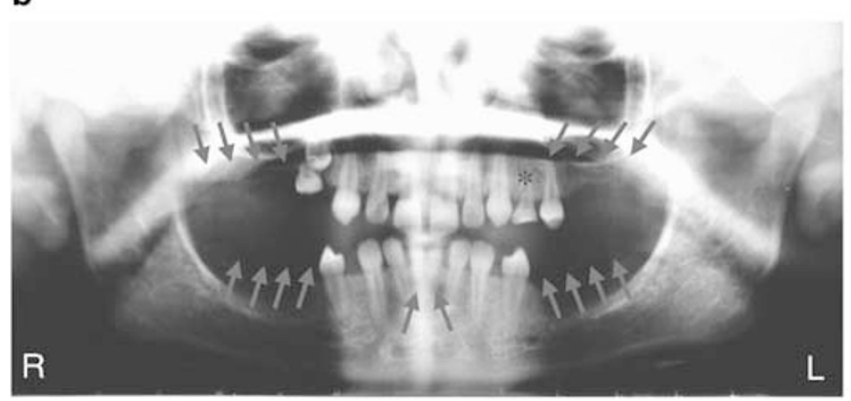

C

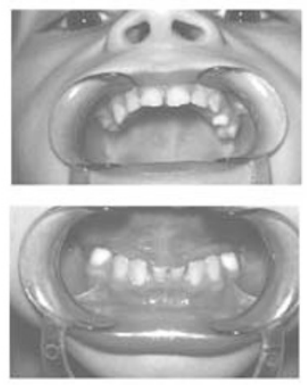

d

\begin{tabular}{|c|c|c|c|c|c|c|c|c|c|c|c|c|c|c|c|c|}
\hline & 18 & 17 & 16 & 15 & 14 & 13 & 12 & 11 & 21 & 22 & 23 & 24 & 25 & 26 & 27 & 28 \\
\hline & 48 & 47 & 46 & 45 & 44 & 43 & 42 & 41 & 31 & 32 & 33 & 34 & 35 & 36 & 37 & 38 \\
\hline 11 & $x$ & $x$ & $x$ & $\mathrm{X}$ & & & & & & & & & $x$ & $x$ & $x$ & $x$ \\
\hline & $x$ & $x$ & $x$ & $x$ & & & & $x$ & $x$ & & & & $x$ & $x$ & $x$ & $x$ \\
\hline Il:2 & $X$ & $x$ & $X$ & $x$ & & & & & & & & & $X$ & $x$ & $x$ & $x$ \\
\hline & $x$ & $x$ & $x$ & $x$ & & & & $x$ & $x$ & & & & $x$ & $\hat{x}$ & $x$ & $x$ \\
\hline II:4 & $\begin{array}{l}X \\
x\end{array}$ & $\begin{array}{l}X \\
x\end{array}$ & $\begin{array}{l}x \\
x\end{array}$ & $\begin{array}{l}x \\
x\end{array}$ & & & & $x$ & $x$ & & & & $\begin{array}{l}X \\
x\end{array}$ & $\begin{array}{l}x \\
x\end{array}$ & $\begin{array}{l}x \\
x\end{array}$ & $\begin{array}{l}X \\
x\end{array}$ \\
\hline & $x$ & $x$ & $x$ & $x$ & & & & & & & & & $x$ & $x$ & $x$ & $\hat{x}$ \\
\hline III:1 & $\hat{x}$ & $\hat{x}$ & $\hat{x}$ & $\hat{x}$ & & & & $x$ & $x$ & & & & $\hat{x}$ & $\hat{x}$ & $\hat{x}$ & $\hat{x}$ \\
\hline & $x$ & $x$ & $x$ & $x$ & & & & & & & & & $x$ & $x$ & $x$ & $x$ \\
\hline III:2 & $\hat{x}$ & $\hat{x}$ & $\hat{x}$ & $\hat{x}$ & & & & $x$ & $x$ & & & & $\hat{x}$ & $\hat{x}$ & $\hat{x}$ & $\hat{x}$ \\
\hline
\end{tabular}

Figure 1 Clinical presentations. (a) Pedigree analysis. Squares, males; circles, females; darkened, affected; (/), deceased; arrow indicates proband. (b) Panoramic radiograph of the proband's dentition (III:1) at the age of 12 years. Arrows indicate missing permanent teeth. Asterisk indicates a preserved primary tooth 63. Eruption of tooth 14 was slightly delayed. (c) Clinical photographs of the proband. 1, upper jaw; 2, lower jaw. (d) Phenotypes of the affected family members. The FDI numbering system was used to designate missing tooth. ( $\mathrm{x}$ ), congenitally missing tooth.

The patterns of missing teeth were identical. All of these individuals lacked molars, second premolars and lower first incisors (Figure $1 \mathrm{~b}-\mathrm{d}$ ). All reported individuals had normal primary dentition and did not reveal abnormalities of nails, skin, hair or sweat glands.

\section{Mutation analysis}

MSSCP analysis of the amplified fragment PAX9ex2C in proband with oligodontia showed an abnormal mobility of single- as well as double-stranded DNA fragments (Figure 2a). Sequence analysis of the separated alleles revealed a novel heterozygous mutation 619_621delAT-
CinsTACCGACCAAGGTAGGGCATCCCT located 13 bp upstream of the $3^{\prime}$ end of exon 2, beyond the highly conserved paired box sequence (Figure 2b). PAGE analysis of the amplified fragment revealed that the mutation was present not only in the proband but also in all affected family members. In the healthy members of the analyzed family, as well as in 150 unrelated control individuals, this mutation was not detected (Figure 2c). The mutation might result either in premature termination of translation at aa 210 or might be a cause of an aberrant splicing, leading to a frameshift and premature termination of translation at aa 314 (Figure 3a and b). The probability was 
a

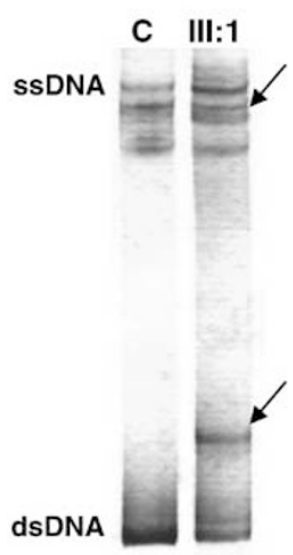

b Normal allele

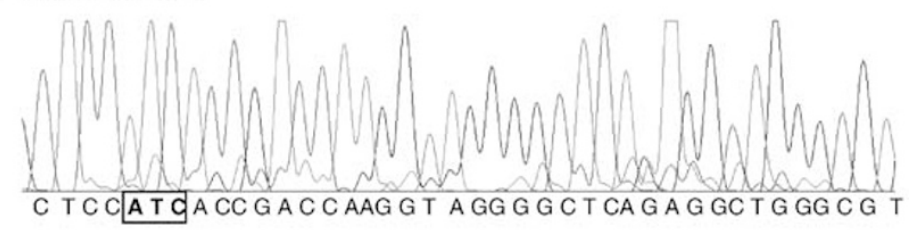

Mutated allele

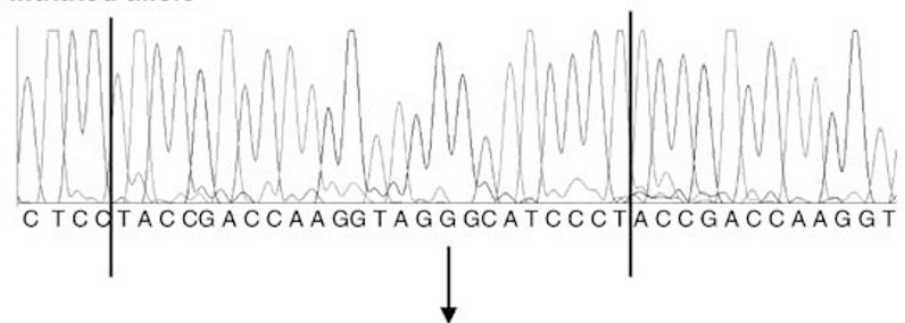

619_621delATCinsTACCGACCAAGGTAGGGCATCCCT

C

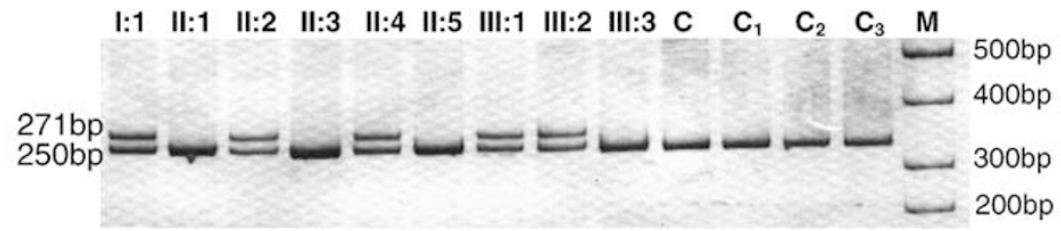

Figure 2 Detection of mutation in exon 2 of PAX9. (a) Multi-temperature, single-stranded conformational polymorphism analysis. C, control; III:1, proband. Arrows show an abnormal MSSCP pattern. (b) Chromatogram showing a novel mutation 619_621delATCinsTACCG ACCAAGGTAGGGCATCCCT. All affected family members were heterozygous for this mutation. (c) Segregation of the mutation. Amplified fragment PAX9ex2C was subjected to polyacrylamide gel electrophoresis in $10 \%$ gel. Normal allele, $350 \mathrm{bp}$; mutated allele $371 \mathrm{bp} ; \mathrm{C}_{1} \mathrm{C}_{1}, \mathrm{C}_{2}, \mathrm{C}_{3}$, controls; $\mathrm{M}$, molecular size marker.

0.97 and 0.95, respectively (http://www.fruitfly.org/seq_ tools/splice.html).

No other mutations in the coding regions of the analyzed genes were found. However, we identified four known polymorphisms of MSX1: 452-15delT, *6C $>\mathrm{T}$ and of $P A X 9: 717 \mathrm{C}>\mathrm{T}, 718 \mathrm{G}>\mathrm{C}$. Analysis of these sequence variants with the use of RFLP and sequencing analyses revealed that they were not associated with oligodontia in the investigated family, since they were present both in the affected as well as in unaffected individuals (results not shown).

\section{Analysis of PAX9 cDNA}

Sequence analysis of the cDNA region corresponding to exons 2 and 3 of $P A X 9$, in the proband as well as in the control revealed normal nucleotide sequence (Figure $4 \mathrm{a}$ ). These results were confirmed by the analysis of melting temperature of the PCR products with the use of LightCycler system (Figure 4b). Analysis of the first negative derivatives of the melting curves showed that the same specific target sequence existed in both proband and control cDNA. In the affected individual heterozygous for the novel deletion combined with insertion, the mutated cDNA sequence was not detected.

\section{Discussion}

Our results strongly suggest that the phenotype of all affected individuals of a three generation family with severe oligodontia was caused by the novel mutation of PAX9, 619_621delATCinsTACCGACCAAGGTAGGGCATCCCT. Since this mutation is not only a deletion combined with an insertion but also a duplication of the sequence containing the $5^{\prime}$ splicing site, its effect on the protein product strongly depends on splicing process of PAX9 premRNA.

Therefore, this mutation might either be a reason for premature termination of translation at aa 210 , or it might result in an aberrant splicing, leading to a frameshift and premature termination of translation at aa 314 . The analysis of splice sites prediction revealed that the probability of selection of the correct $5^{\prime}$ splice site and the site created by the mutation was almost equal and amounted to 0.97 and 0.95 , respectively.

Regardless of the possible effect of the novel mutation on the splicing process, the structure of functionally important C-terminal region of the encoded transcription factor was considerably perturbed. Incorrect amino-acid sequence of the C-end of PAX9 protein was also described in another large family with oligodontia. ${ }^{4}$ In this case, the insertion of 


\author{
Normal sequence \\ 5' A T C CGCTCCATCACCGACCAAGgtaggggct c agagg ctggg \\ exon $2 \quad 5$ 'splice site intron 2
}

\title{
Mutated sequence

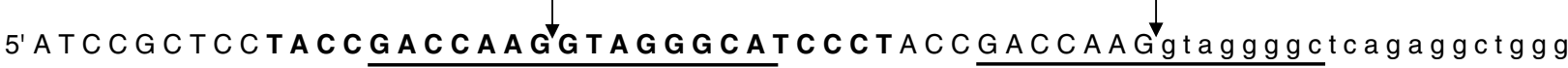 novel 5 ' splice site correct 5 ' splice site
}

b

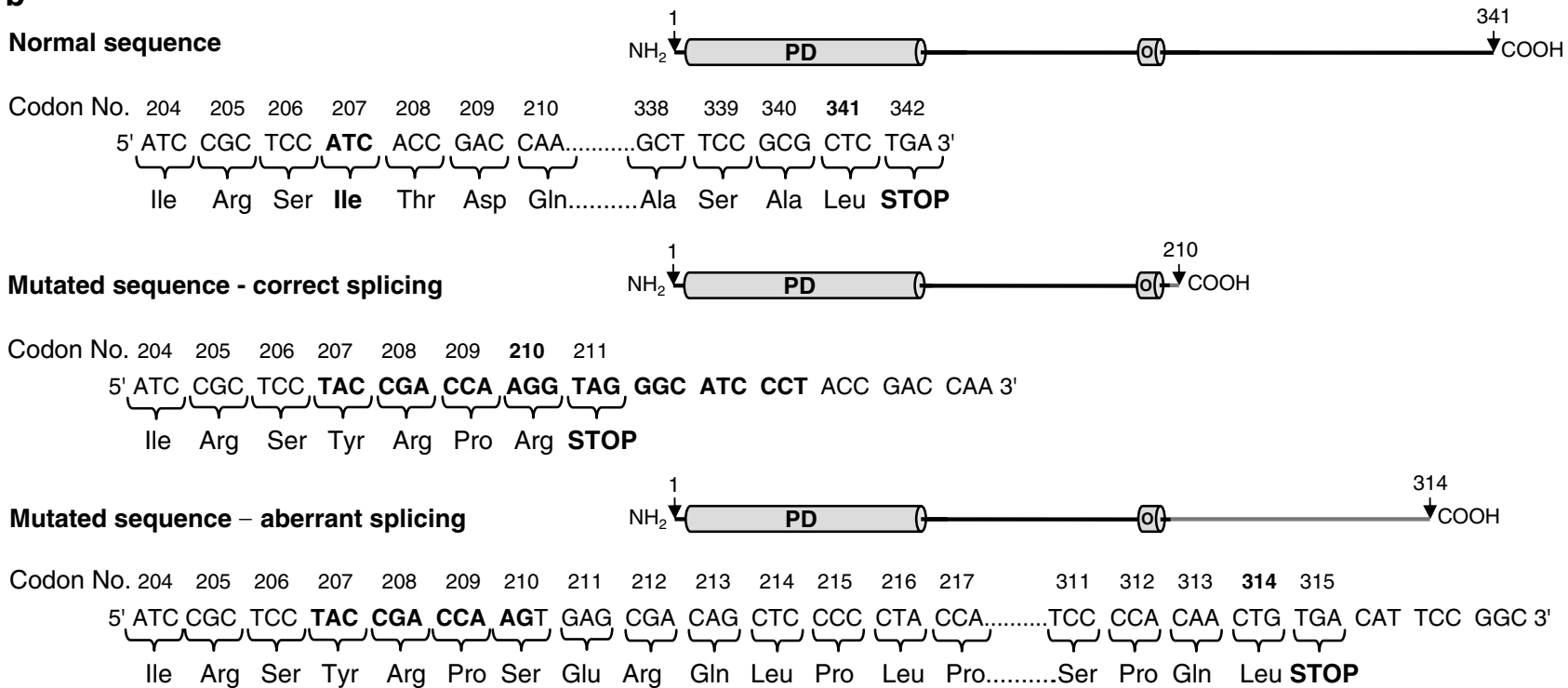

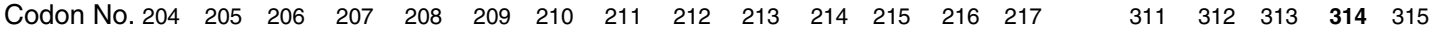

Figure 3 Putative effect of mutation on the structure of the protein product. (a) Comparison of the nucleotide sequences of the normal and mutant gene. The mutation that is not only a deletion combined with insertion but also duplication creates a novel $5^{\prime}$ splice site. Capital letters, exon sequence; small letters, intron sequence; green letters deleted sequence; red letters inserted sequence; splice sites are underlined. (b) Comparison of nucleotide and amino-acid sequences of normal and mutant mRNAs and proteins. Schematic diagrams of wild-type and mutated protein structures are included. PD, paired domain; O, octapeptide.

additional $\mathrm{G}$ within the paired box sequence at nucleotide 219 resulted in a frameshift and premature termination of translation 243 codons downstream of the insertion. Functional analysis of the mutant protein revealed dramatically reduced ability to activate transcription. ${ }^{22}$ This inactivating mutation impairs the interaction and activation of the transcription factor recognition sequences on the promotores/enhancers of target genes. In addition, immunolocalization data showed an altered cellular localization of the mutated protein, which appeared to form aggregates in the cytoplasm and was mislocalized to the perinuclear region. ${ }^{22}$ Similar effect of an insertion mutation on PAX9 binding capacity was described by Zhao et $a .^{28}$ They identified a novel mutation located in paired box sequence (109insG) resulting in the loss of function of the encoded transcription factor. On the basis of these results, we speculate that our novel mutation might reduce transactivation activity of PAX9 protein, and might be responsible for oligodontia.

In order to analyze the effect of $P A X 9$ deletion combined with insertion on splicing process, the cDNA obtained from cultured lymphocytes of the affected individual harboring this mutation was sequenced as well as analyzed by real-time PCR. Both analyses revealed only the normal cDNA sequence, whereas the mutated sequence was not detected. These results confirmed previous reports that in the living cells exists a very important quality control mechanism, reducing the amount of nonfunctional mRNAs that might produce truncated proteins. This nonsense-mediated decay (NMD) mechanism of eliminating mRNAs containing mutations that introduce premature termination codons is a common finding in many diseases. ${ }^{29,30}$ The effect of the novel mutation of $P A X 9$ on its mRNA content fulfils requirement of the NMD 
associated with molar agenesis. ${ }^{7,31}$ The effect of haploin-

a III:1

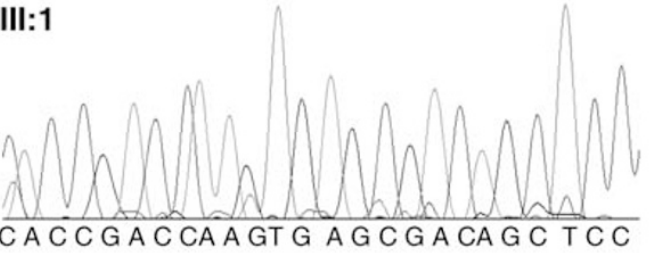

Control
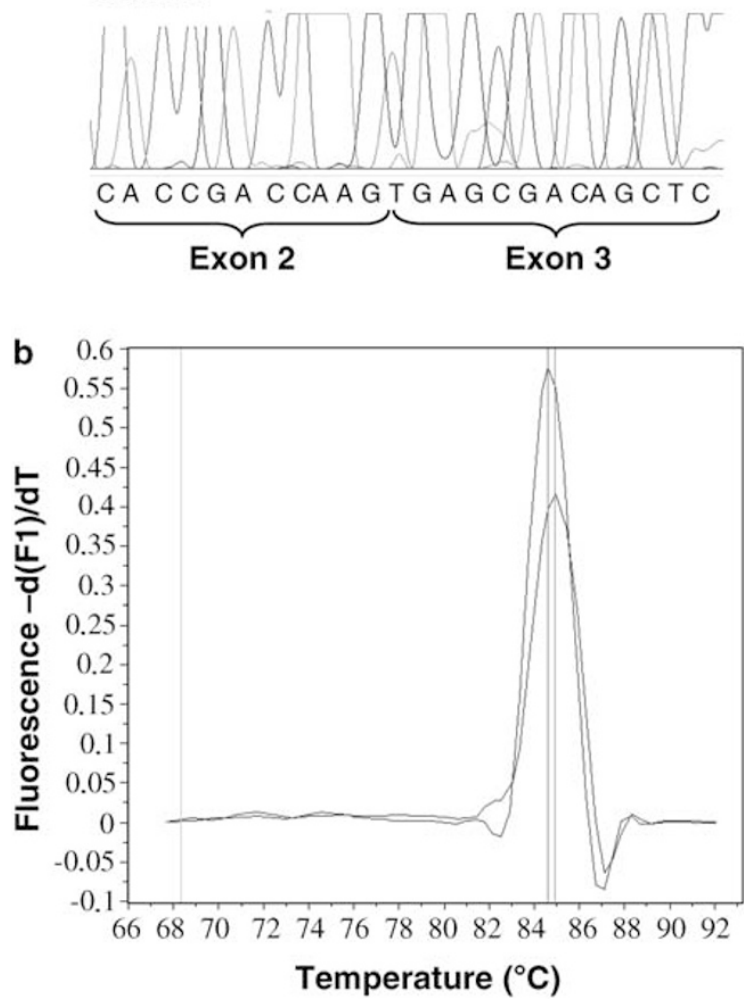

Figure 4 Analysis of PAX9 CDNA. (a) Sequence analysis of the region corresponding to the second and the third exon of PAX9 revealed the presence of normal CDNA sequence in both the proband and the control. (b) Melting curve profiles. Analysis of the first negative derivatives of the melting curves $(-\mathrm{dF} / \mathrm{dT})$ clearly showed that the same specific target sequence existed in both samples. Characteristic melting temperature was approximately $84.5^{\circ} \mathrm{C}$.

mechanism, suggesting that the absence of mutated cDNA sequence might result from rapid mRNA degradation. However, functional assay is required to confirm this hypothesis.

In conclusion, the novel heterozygous mutation, like all other mutations responsible for tooth agenesis, might exert its phenotypic effect through inactivation of one copy of the protein. However, in the case of the analyzed mutation, haploinsufficiency might be caused either by reduction of transactivation capacity of PAX9 protein mediated by Cterminal amino acids, or by rapid degradation of mutated PAX9 mRNA via the nonsense-mediated mRNA decay mechanism.

The phenotypes of all affected family members confirmed the view that PAX9 mutations are dominantly sufficiency of PAX9 on the development of incisors and premolars is probably caused by deficiency of MSX1, whose mutations mainly affect the development of these teeth. ${ }^{7}$ It was demonstrated that at the bud stage, the expression of Msx 1 in knockout mice homozygous for $\operatorname{Pax} 9$ deletion is substantially downregulated. ${ }^{20}$ In addition, PAX9 mutation might affect normal interactions between PAX9 and MSX1 that play a very important role in the early events underlying odontogenesis as suggested by Ogawa et al. ${ }^{32}$

Despite many years of investigations concerning the molecular processes governing odontogenesis, our knowledge on molecular basis of the most common developmental abnormalities of human dentition remains obscure. Although PAX9 is one of the main candidate genes, whose mutations might be responsible for selective tooth agenesis, they were found only in a small number of cases. It is interesting to note that the most recent study has indicated that polymorphisms localized in the promotor region of PAX9 might also be associated with hypodontia. ${ }^{33}$ This observation throw a new light on etiology of selective tooth agenesis, suggesting that not only mutations but also polymorphic variants of main candidate genes might be a risk factor for this anomaly.

\section{Acknowledgements}

This work was supported by Grant No. P05A 09226 from the State Committee for Scientific Research (KBN). Editorial assistance of $\mathrm{Mr}$ Robert Meehan (M.Sc.) is gratefully acknowledged.

\section{References}

1 Vastardis $\mathrm{H}$ : The genetics of human tooth agenesis: new discoveries for understanding dental anomalies. Am J Orthod Dentofacial Orthop 2000; 117: 650-656.

2 Schalk-van der Weide Y, Beemer FA, Faber JA, Bosman F: Symptomatology of patients with oligodontia. I Oral Rehabil 1994; 21: $247-261$.

3 Vastardis H, Karimbux N, Guthua SW, Seidman JG, Seidman CE: A human MSX1 homeodomain missense mutation causes selective tooth agenesis. Nat Genet 1996; 13: 417-421.

4 Stockton DW, Das P, Goldenberg M, D'Souza RN, Patel PI: Mutation of PAX9 is associated with oligodontia. Nat Genet 2000; 24: 18-19.

5 van den Boogaard MJ, Dorland M, Beemer FA, van Amstel HK: MSX1 mutation is associated with orofacial clefting and tooth agenesis in humans. Nat Genet 2000; 24: 342-343.

6 Jumlongras D, Bei M, Stimson JM et al: A nonsense mutation in MSX1 causes Witkop syndrome. Am J Hum Genet 2001; 69: $67-74$.

7 Nieminen P, Arte S, Tanner D et al: Identification of a nonsense mutation in the PAX9 gene in molar oligodontia. Eur J Hum Genet 2001; 9: 743-746.

8 Das P, Stockton DW, Bauer C et al: Haploinsufficiency of PAX9 is associated with autosomal dominant hypodontia. Hum Genet 2002; 110: 371-376.

9 Frazier-Bowers SA, Guo DC, Cavender A et al: A novel mutation in human PAX9 causes molar oligodontia. J Dent Res 2002; 81: $129-133$.

10 Lidral AC, Reising BC: The role of MSX1 in human tooth agenesis. J Dent Res 2002; 81: 274-278. 
11 Das $\mathrm{P}$, Hai $\mathrm{M}$, Elcock $\mathrm{C}$ et al: Novel missense mutations and a 288-bp exonic insertion in PAX9 in families with autosomal dominant hypodontia. Am J Med Genet 2003; 118: $35-42$.

12 Lammi L, Halonen K, Pirinen S, Thesleff I, Arte S, Nieminen P: A missense mutation in PAX9 in a family with distinct phenotype of oligodontia. Eur J Hum Genet 2003; 11: 866-871.

13 Mostowska A, Kobielak A, Biedziak B, Trzeciak WH: Novel mutation in the paired box sequence of PAX9 gene in a sporadic form of oligodontia. Eur J Oral Sci 2003; 111: 272-276.

14 De Muynck S, Schollen E, Matthijs G, Verdonck A, Devriendt K, Carels C: A novel MSX1 mutation in hypodontia. Am J Med Genet 2004; 128: 401-403.

15 Jumlongras D, Lin JY, Chapra A et al: A novel missense mutation in the paired domain of PAX9 causes non-syndromic oligodontia. Hum Genet 2004; 114: 242-249.

16 Klein ML, Nieminen P, Lammi L, Niebuhr E, Kreiborg S: Novel mutation of the initiation codon of PAX9 causes oligodontia. J Dent Res 2005; 84: 43-47.

17 Tucker AS, Al Khamis A, Sharpe PT: Interactions between Bmp-4 and Msx-1 act to restrict gene expression to odontogenic mesenchyme. Dev Dyn 1998; 212: 533-539.

18 Peters H, Balling R: Teeth. Where and how to make them. Trends Genet 1999; 15: 59-65.

19 Satokata I, Maas R: Msx1 deficient mice exhibit cleft palate and abnormalities of craniofacial and tooth development. Nat Genet 1994; 6: 348-356.

20 Peters H, Neubuser A, Kratochwil K, Balling R: Pax9-deficient mice lack pharyngeal pouch derivatives and teeth and exhibit craniofacial and limb abnormalities. Genes Dev 1998; 12: $2735-2747$.

$21 \mathrm{Hu}$ G, Vastardis H, Bendall AJ et al: Haploinsufficiency of MSX1: a mechanism for selective tooth agenesis. Mol Cell Biol 1998; 18: 6044-6051.

22 Mensah JK, Ogawa T, Kapadia H, Cavender AC, D'Souza RN: Functional analysis of a mutation in PAX9 associated with familial tooth agenesis in humans. I Biol Chem 2004; 279: $5924-5933$

23 Jho EH, Zhang T, Domon C, Joo CK, Freund JN, Costantini F: Wnt/beta-catenin/Tcf signaling induces the transcription of Axin2, a negative regulator of the signaling pathway. Mol Cell Biol 2002; 22: 1172-1183.

24 Liu W, Dong X, Mai M et al: Mutations in AXIN2 cause colorectal cancer with defective mismatch repair by activating beta-catenin/ TCF signalling. Nat Genet 2000; 26: 146-147.

25 Lammi L, Arte S, Somer M et al: Mutations in AXIN2 cause familial tooth agenesis and predispose to colorectal cancer. $\mathrm{Am} \mathrm{J}$ Hum Genet 2004; 74: 1043-1050.

26 Vieira AR, Meira R, Modesto A, Murray JC: MSX1, PAX9 and TGFA contribute to tooth agenesis in humans. J Dent Res 2004; 83: $723-727$.

27 Lidral AC, Romitti PA, Basart AM et al: Association of MSX1 and TGF $\beta 3$ with nonsyndromic clefting in humans. Am J Hum Genet 1998; 63: 557-568.

28 Zhao JL, Chen YX, Bao L et al: Novel mutations of PAX9 gene in Chinese patients with oligodontia. Zhonghua Kou Qiang Yi Xue Za Zhi 2005; 40: 266-270.

29 Frischmeyer PA, Dietz HC: Nonsense-mediated mRNA decay in health and disease. Hum Mol Genet 1999; 8: 1893-1900.

30 Bateman JF, Freddi S, Nattrass G, Savarirayan R: Tissue-specific RNA surveillance? Nonsense-mediated mRNA decay causes collagen X haploinsufficiency in Schmid metaphyseal chondrodysplasia cartilage. Hum Mol Genet 2003; 12: 217-225.

31 Mostowska A, Kobielak A, Trzeciak WH: Molecular basis of nonsyndromic tooth agenesis: mutations of MSX1 and PAX9 reflect their role in patterning human dentition. Eur J Oral Sci 2003; 111: $365-370$.

32 Ogawa T, Kapadia H, Wang B, D'Souza RN: Studies on Pax9-Msx1 protein interactions. Arch Oral Biol 2005; 50: 141-145.

33 Peres RC, Scarel-Caminaga RM, do Espirito Santo AR, Line SR: Association between PAX-9 promoter polymorphisms and hypodontia in humans. Arch Oral Biol 2005; 50: 861-871. 